\title{
Effets de la pollinisation entomophile sur le colza dans une expérience en cage
}

\author{
Jacques LERIN \\ avec la collaboration technique d'Eric RIVAULT \\ I.N.R.A., Laboratoire de Zoologie, F86600 Lusignan.
}

\section{RÉSUMÉ}

Pollinisation entomophile, Brassica napus var. oleifera, Bombus terrestris, Composantes du rendement, Compensation,

Essai sous cage.

\begin{abstract}
On a étudié les effets de la pollinisation du colza (Brassica napus L. var. oleifera Metzger) par un bourdon (Bombus terrestris L.) dans une expérience en cage, en vue de déterminer les conditions les plus favorables à une production de graines homogène par les plantes. Cette homogénéisation est une nécessité pour l'étude des pertes de rendements dues à un ravageur.

La pollinisation entomophile augmente considérablement la production de l'inflorescence principalc par accroissement du nombre de siliques et du nombre de graines par silique. Ceci entraîne au niveau de la plante entière, une diminution du nombre d'inflorescences, notamment tertiaires. L'étude des inflorescences de même rang suggère l'existence d'une inhibition de l'initiation florale ou du développement des siliques lorsque la production de la hampe principale est importante. Le drainage des métabolites par l'inflorescence principale entraîne une réduction du nombre de graines par silique sur les inflorescences secondaires les plus hautes. Cet effet disparait pratiquement au niveau de la $4^{\mathrm{c}}$ inflorescence secondaire. Au niveau de la plante entière la pollinisation a un effet essentiellement qualitatif : elle réduit la variabilité des composantes du rendement et homogénéise la maturation et la production. L'augmentation de $26 \mathrm{p} .100 \mathrm{du}$ rendement des plantes entières pollinisées par rapport aux non pollinisées n'est pas significative.
\end{abstract}

\section{Effects of pollination on rape in a cage experiment}

The effects of pollination of rape (Brassica napus L. var. oleifera Metzger) by a bumble bee (Bombus terrestris L.) have been studied in a cage experiment. The principal aim was to find out the best conditions for ensuring homogeneous yicld. This is required for studies on yield losses caused by pests. Pollination greatly increased the yield of the main shoot through an increase in the number of pods and number of seeds per pod. This led to a reduced number of racemes per plant. The study of racemes at the same level suggests that a high-yielding main shoot has an inhibitory effect on floral initiation and pod development. The draining of metabolites by the main shoot induces a reduced number of seeds per pod on the highest secondary racemes. This effect practically disappears at the level of the 4th secondary raceme. At the whole plant level insect pollination has an essentially qualitative effect : it reduces variability among yield components so that maturity and yield components are more homogeneous. The $26 \%$ increase in yield in pollinated plants (compared with unpollinated ones) is not statistically significant.

\section{INTRODUCTION}

Nous avons réalisé une étude méthodologique afin de déterminer les conditions dans lesquelles les plantes de colza (Brassica napus L. var. oleifera Metzger) en cage ont la production la plus homogène possible. Cette recherche s'avère un préliminaire indispensable pour étudier les pertes de rendement dues à certains ravageurs. A l'heure actuelle il est en effet impossible d'utiliser d'autre méthode que la cage pour, à la fois, maintenir un nombre donné d'une espèce de ravageur et exclure tous les autres.

Ces cages ont un effet brise-vent évident et les plantes y sont très peu agitées; dans le modèle expérimental choisi elles sont également peu denses à l'unité de surface: les frottements entre inflorescences sont donc limités et l'absence de colza à l'extérieur des cages réduit la probabilité d'une pollinisation anémophile. Les conditions hygro- thermiques régnant à l'intérieur de la cage peuvent également entraîner une mauvaise déhiscence des anthères. On se rapproche ainsi des conditions de serre.

Or une observation fortuite réalisée l'an dernier sur le nombre de siliques par unité de longueur sur les inflorescences de colza dans des serres où étaient élevés des bourdons a suggéré un effet marqué de la pollinisation par ces insectes. Il nous est donc apparu nécessaire pour notre méthodologie de quantifier l'importance de la pollinisation entomophile du colza.

Cette plante étant autogame à 70 p. 100 (RIvEs, 1957) et les variétés actuellement cultivées en France étant des lignées pures (RENARD com. pers.) l'effet, a priori stimulant, d'une pollinisation croisée devrait être nul à l'intérieur d'une même variété. C'est pourquoi les nombreux résultats publiés montrant un effet bénéfique de la pollinisation sont discutés (BARBIER, 1978 ; EISIKOWITCH, 1981 ; MESQUIDA 
\& Renard, sous presse). Free \& Nuttall (1968) mentionnent plusieurs publications (remontant jusqu'en 1939) où tous les auteurs constatent des augmentations de rendement de 17 à 55 p. 100 sur des plantes, en cage, pollinisées par des abeilles par rapport à des plantes non pollinisées. Ces expériences étaient faites sans répétition et, depuis, les variétés ont été profondément modifiées par sélection. L'expérimentation de ces deux auteurs met en évidence une augmentation non significative de 13 p. 100 du nombre de graines par plante dans le traitement "pollinisé sous cage " par rapport au traitement "non pollinisé sous cage ». En revanche le poids de 1000 graines est significativement plus élevé sur les plantes non pollinisées. On se trouve ainsi en présence d'une augmentation du nombre de graines par plante insuffisante pour devenir statistiquement significative mais suffisante pour entrainer une modification du poids de 1000 graines. Tous les auteurs mentionnant les composantes du rendement du colza signalent également une augmentation du nombre de graines, compensée en partie par une diminution de leur poids, à l'exception de BENEDEK et al. (1972) qui observent une augmentation du poids de 1000 graines dans le lot pollinisé.

WiLliaMs (1978) a montré que le nombre de graines par silique et l'aptitude à former des siliques différaient suivant les variétés de colza et le mode de pollinisation dans une expérience en serre : la pollinisation manuelle croisée entre plantes différentes de même cultivar et la pollinisation avec du pollen de la même fleur donnent des résultats similaires ; en revanche l'autopollinisation (sans aide extérieure) donne des résultats inféricurs aux deux précédents. Dans un $4^{\mathrm{c}}$ traitement, des plantes ont été secouées quotidiennement pour simuler l'action du vent et ont donné un nombre de siliques et un nombre de graines par silique intermédiaire. L'auteur note également une réduction de la durée de floraison dans les lots pollinisés à la main.

Les phénomènes de compensation au niveau de chaque plante chez les Brassica cultivés ont été très souvent invoqués pour expliquer l'absence de différence significative mais en fait très peu étudiés. C'est pourquoi il nous a paru nécessaire d'examiner la production des plantes par catégoric d'inflorescences.

\section{MATÉRIEL ET MÉTHODES}

Le dispositif comprenait 4 cages de $8 \mathrm{~m}^{3}$ recouvertes de grillage à mailles fines (14 mailles au $\mathrm{cm}$ ) contenant chacune 8 caissettes en bois remplies d'un mélange de sable, de terre franche et de terreau en parties égales. Chaque caissette $(30 \times 10 \times 15 \mathrm{~cm})$ contenait 2 pieds de colza, variété « Jet neuf ", soit 16 plantes par cage. Deux cages ont reçu une fondatrice de bourdon (Bombus terrestris L.), prélevée dans la nature avant que la colonie ne soit fondée, en début de floraison (le 25 avril 1980). Ces bourdons ont été retirés à la fin de la floraison du lot pollinisé (le 21 mai 1980). Quelques plantes sans inflorescence principale présentant une pourriture du bourgeon terminal et un port buissonnant ont été éliminées des résultats ( 3 dans le lot non pollinisé, 1 dans le lot pollinisé).

Les inflorescences ont ćté prélevées une à une au fur et à mesure qu'elles arrivaient à maturité. Pour chaque plante on a relevé lc diamètre au collet (D.C.), le nombre d'inflorescences productives, ayant au moins 2 siliques (N.I.), la longueur de l'inflorescence principale (long.) entre la $1^{\text {re }}$ et la dernière silique productive.

Sur chaque inflorescence (inflorescence principale I.P., inflorescences secondaires $I_{1}, I S_{2}, I_{3} \ldots$ selon l'ordre d'apparition), les caractéristiques suivantes ont été relevées:

- $\mathrm{Sx}$ : nombre de siliques productives (portant au moins une graine),

- EG/Sx : estimation du nombre de graines par silique déterminé sur 10 siliques prélevées au hasard,

- Gx: nombre total de graines de l'inflorescence,

- Px : poids de graines (en $\mathrm{g}$ ) après passage à l'étuve à $60 \pm 2{ }^{\circ} \mathrm{C}$ pendant $48 \mathrm{~h}$.

Cette analyse a été faite pour chaque inflorescence prise individuellement, de la hampe primaire à la 5 inflorescence secondaire, les inflorescences suivantes étant analysées globalement (inflorescences secondaires restantes: ISR).

Les résultats ont été regroupés pour chaque plante en 3 séries de variables ayant trait respectivement à l'inflorescence principale (notée IP), à la somme des inflorescences secondaires (IS), au total par plante (T).

Le nombre réel de graines par silique $\mathrm{G} / \mathrm{Sx}$ a été calculé en divisant le nombre de graines par le nombre de siliques; de même pour le poids moyen d'une graine $\mathrm{Pgx}=\mathrm{Px} / \mathrm{Gx}$ exprimé en grammes.

Toutes les données ont été transformées en $\log _{c}$ sauf « D.C. », « NI » et « long. ». Les résidus de la régression du rendement par plante $\left(\mathrm{P}_{\mathrm{T}}\right)$ en fonction des 3 composantes qui le déterminent (nombre de siliques, graines par silique, poids d'une graine) augmentent avec les valeurs de $\mathrm{P}_{\mathrm{T}}$. La transformation logarithmique élimine cet effet et homogénéise les variances. Les moyennes des différentes variables mesurées n'étant pas statistiquement différentes entre cages de même traitement, les comparaisons entre lot pollinisé et lot non pollinisé ont été faites sur l'ensemble des plantes de chaque traitement, soit 29 pour les plantes sans bourdon et 31 pour les plantes avec bourdon.

Pour faciliter la lecture des tableaux, les moyennes établies sur les données transformées en $\log _{e}$ ont été retranscrites en valeurs réelles mais les variances sont celles des moyennes exprimées en $\log _{c}$.

\section{RÉSULTATS - DISCUSSION}

\section{A. Observations}

Les estimations du nombre de graines par silique (EG/Sx) fournissent des résultats imprécis par rapport au calcul du nombre réel $(\mathrm{Gx} / \mathrm{Sx})$. En particulier le pourcentage de la variance du nombre moyen de graines par silique sur la plante entière $\left(\mathrm{G} / \mathrm{S}_{\mathrm{T}}\right)$, expliqué par son estimation $\left(\mathrm{EG} / \mathrm{S}_{\mathrm{T}}\right)$, est au maximum de $73 \mathrm{p}$. 100. Il n'en sera question par la suite qu'au moment de la comparaison avec les plantes prélevées au champ où l'estimation était la seule méthode praticable, les comptages ayant été faits sur des plantes encore vertes pour éviter l'égrenage.

Au moment du retrait des bourdons, pour l'ensemble des plantes pollinisées, seules quelques inflorescences portaient encore des fleurs ouvertes. En revanche, dans le lot non pollinisé la floraison continuait sur toutes les plantes sur des inflorescences «tertiaires » pratiquement inexistantes dans le lot pollinisé. A la récolte, la production de ces dernières a été englobée dans la production des "secondaires" les portant, faute de moyens suffisants pour les individualiser. La maturation des plantes était donc beaucoup plus hétérogène dans le lot non pollinisé et, sur les dernières inflorescences tertiaires, des siliques encore vertes contenant des graines brun-rouge contrastaient avec les siliques mûres des extrémités des inflorescences secondaires. 


\section{B. Inflorescences principales (tabl. 1 et 2)}

C'est sur les inflorescences principales que l'effet de la pollinisation entomophile est le plus marqué : il y a une augmentation du nombre de siliques et du nombre de graines par silique. L'augmentation du nombre total de graines qui en résulte se traduit par une diminution des poids de 1000 graines, mais le bilan global de la production est en faveur du lot pollinisé. L'allongement des inflorescences dans le lot non pollinisé traduit une floraison beaucoup plus étalée dans le temps : tout se passe, dans ce cas, comme si la plante produisait des fleurs jusqu'à ce qu'un nombre minimal de graines soit formé, sans pour autant rattraper le lot pollinisé. Dans ce dernier lot, l'inflorescence produit plus du double de graines, ce qui se traduit par une augmentation de rendement de 93 p. 100.

L'analyse des composantes du rendement montre que, dans le lot pollinisé, les variations du nombre de siliques ne déterminent que $23 \mathrm{p} .100$ des variations du nombre de graines alors qu'elles en déterminent 76 p. 100 dans le lot non pollinisé (tabl. 2). La plante ayant une production maximale en nombre de siliques, ce sont les variations du nombre de graines par silique qui deviennent déterminantes dans le lot pollinisé. Ces variations peuvent provenir, soit de différences de pollinisation, soit d'un avortement physiologique, la plante ne pouvant nourrir qu'un certain nombre de graines. Rappelons cependant qu'un tiers des métabolites se retrouvant dans les graines proviennent de l'activité photosynthétique de la paroi des siliques (BRAR \& THIES, 1976) et que la hampe primaire semble drainer une bonne partie des ressources élaborées par la dernière feuille et la partie supérieure de la tige : l'avortement de graines ou de siliques est toujours plus important sur les inflorescences secondaires (CLARKE, 1979). Il faut noter également que toutes les variances sont supérieures dans le lot non pollinisé, de façon significative pour SIP, GIP et PIP ou non pour G/SIP, PgIP, Long. (tabl. 1) : la pollinisation diminue l'hétérogénéité des plantes.

\section{Inflorescences secondaires (tabl. 3, fig. 1)}

L'effet de la pollinisation entomophile n'apparait ici que sur le nombre de graines par silique, le nombre de siliques et le nombre d'inflorescences (tabl. 3). Le déficit de pollinisation est compensé, chez certaines plantes, par une augmentation du nombre de siliques, notamment sur les inflorescences secondaires les plus basses et par l'initiation d'inflorescences supplémentaires. La pollinisation accroît la production par inflorescence de même rang essentiellement par augmentation du nombre de graines par silique (fig. 1). En revanche, il n'y a pas, contrairement à ce qui est constaté sur l'inflorescence principale, d'augmentation significative du nombre de siliques par inflorescence (fig. 1). Au contraire, c'est le lot non pollinisé qui présente le plus de siliques mais aucune différence avec le lot pollinisé n'est significative. Dans le lot pollinisé, le poids moyen de la graine par inflorescence secondaire est inversement corrélé au nombre de graines par silique dans le lot pollinisé

TABLEAU 1

Caractéristiques des inflorescences principales. Characteristics of main shoots.

\begin{tabular}{|c|c|c|c|c|c|c|c|}
\hline & & $\mathrm{Nb}$ siliques & Graines/siliques & $\begin{array}{l}\text { Pds d'1 graine } \\
(\mathrm{g})\end{array}$ & $\begin{array}{l}\text { Rendement } \\
\text { (g) }\end{array}$ & $\begin{array}{l}\text { Longueur } \\
\text { inflorescence } \\
(\mathrm{cm})\end{array}$ & $\mathrm{Nb}$ graines \\
\hline $\begin{array}{l}\text { sans bourdon } \\
\mathrm{n}=29\end{array}$ & $\begin{array}{l}\text { moy. } \\
\text { var. }\end{array}$ & $\begin{array}{l}55,4 \\
0,0889\end{array}$ & $\begin{array}{l}10,02 \\
0,0558\end{array}$ & $\begin{array}{c}7,04.10^{-3} \\
0,0067\end{array}$ & $\begin{array}{l}3,90 \\
0,1773\end{array}$ & $\begin{array}{c}61,3 \\
198,43\end{array}$ & $\begin{array}{r}555,1 \\
0,1978\end{array}$ \\
\hline $\begin{array}{l}\text { avec bourdon } \\
\mathrm{n}=31\end{array}$ & $\begin{array}{l}\text { moy. } \\
\text { var. }\end{array}$ & $\begin{array}{l}65,5 \\
0,0322\end{array}$ & $\begin{array}{l}18,43 \\
0,0446\end{array}$ & $\begin{array}{c}6,23.10^{-3} \\
0,0056\end{array}$ & $\begin{array}{l}7,52 \\
0,0451\end{array}$ & $\begin{array}{c}47,2 \\
112,53\end{array}$ & $\begin{array}{r}1207,2 \\
0,0509\end{array}$ \\
\hline $\begin{array}{l}t \text { ou } t^{\prime} \\
\text { signification }\end{array}$ & & $\begin{array}{c}\text { (') } 2,619 \\
*\end{array}$ & $\begin{array}{c}10,503 \\
* *\end{array}$ & $\begin{array}{c}5,958 \\
* *\end{array}$ & $\begin{array}{ll}\text { (') }^{\prime} & 7,540 \\
& * *\end{array}$ & $\begin{array}{l}4,384 \\
* *\end{array}$ & 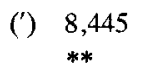 \\
\hline
\end{tabular}

$\mathbf{t}^{\prime}$ : test $\mathbf{t}$ modifié lorsque les variances sont significativement différentes (') (SNEDECOR \& COCHRAN, 1971)

*: seuil à 5 p. 100

**: : seuil à 1 p. 100

TABLEAU 2

Corrélations entre des composantes du rendement des inflorescences principales. Correlations between some yield components on the main shoots.

\begin{tabular}{lcccc}
\hline \hline & pollinisé & $\begin{array}{c}\text { Nb de siliques } \\
\text { non pollinisé }\end{array}$ & pollinisé & $\begin{array}{c}\text { Nb de graines } \\
\text { non pollinisé }\end{array}$ \\
\hline Nb de graines & 0,475 & 0,871 & 1,000 \\
\hline Rendement & 0,477 & 0,851 & 0,943 & 0,984 \\
\hline \hline
\end{tabular}


TABLEAU 3

Caractéristiques des inflorescences secondaires.

Characteristics of secondary racemes.

\begin{tabular}{|c|c|c|c|c|c|c|c|}
\hline & & $\mathrm{Nb}$ siliques & Graines/siliques & $\begin{array}{l}\text { Pds d'1 graine } \\
\text { (g) }\end{array}$ & $\begin{array}{l}\text { Rendement } \\
(\mathrm{g})\end{array}$ & $\begin{array}{c}\mathrm{Nb} \\
\text { d'inflorescences }\end{array}$ & $\begin{array}{c}\mathrm{Nb} \\
\text { de graines }\end{array}$ \\
\hline sans bourdon & $\begin{array}{l}\text { moy. } \\
\text { var. }\end{array}$ & $\begin{array}{l}274,1 \\
0,3315\end{array}$ & $\begin{array}{c}10,36 \\
0,0790\end{array}$ & $\begin{array}{c}6,34 \cdot 10^{-3} \\
0,0122\end{array}$ & $\begin{array}{c}18,00 \\
0,4314\end{array}$ & $\begin{array}{l}9,17 \\
4,22\end{array}$ & $\begin{array}{r}2838,4 \\
0,5201\end{array}$ \\
\hline avec bourdon & $\begin{array}{l}\text { moy. } \\
\text { var. }\end{array}$ & $\begin{array}{l}209,9 \\
0,0997\end{array}$ & $\begin{array}{c}15,70 \\
0,0546\end{array}$ & $\begin{array}{c}6,09 \cdot 10^{-3} \\
0,0063\end{array}$ & $\begin{array}{l}20,06 \\
0,1840\end{array}$ & $\begin{array}{l}7,90 \\
1,69\end{array}$ & $\begin{array}{r}3296,4 \\
0,2016\end{array}$ \\
\hline $\begin{array}{l}t \text { ou } t^{\prime} \\
\text { signification }\end{array}$ & & $\begin{array}{c}\text { (') } 2,204 \\
*\end{array}$ & $\begin{array}{c}6,357 \\
* *\end{array}$ & $\begin{array}{l}\text { (') } 1,611 \\
\mathrm{NS}\end{array}$ & $\begin{array}{l}\text { (') } 0,751 \\
\text { NS }\end{array}$ & (') $\begin{array}{l}2,840 \\
*\end{array}$ & $\begin{array}{l}\text { (') } 0,957 \\
\text { NS }\end{array}$ \\
\hline
\end{tabular}

( $r=-0,94$ significatif à 1 p. 100 pour 4 d.1.). Cette relation est non significative $(r=-0,53)$ dans le lot non pollinisé.

Les phénomènes de compensation au niveau de chaque plante entre l'inflorescence principale et les inflorescences secondaires sont doubles. Dans le lot pollinisé, ils apparaissent nettement : ils jouent sur le nombre de siliques formées sur la $1^{\text {rc }}$ inflorescence secondaire et dans une moindre mesure sur la $2^{\mathrm{c}}$ ainsi que sur le nombre de graines par

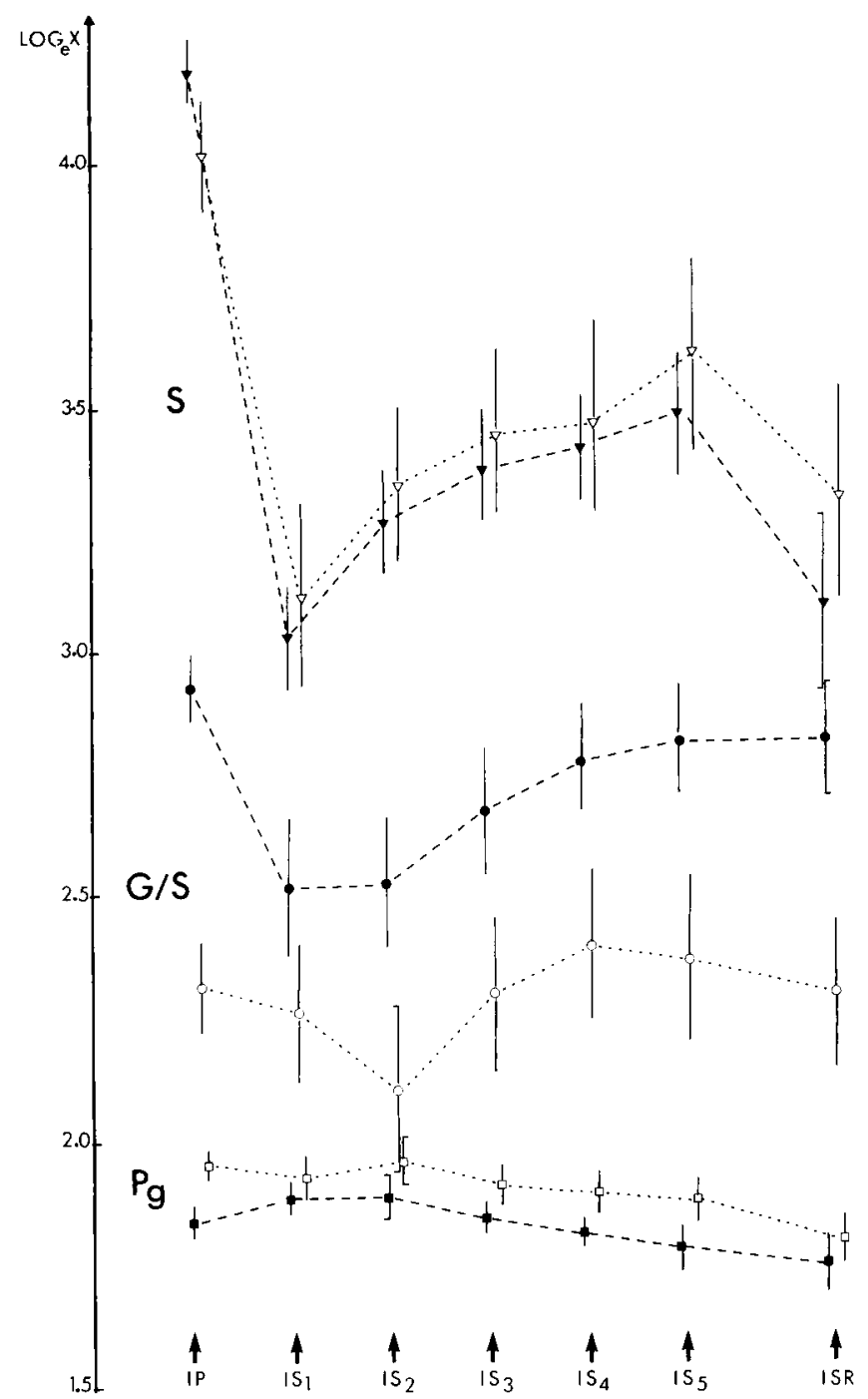

silique pouvant se développer sur les 3 premières inflorescences secondaires. Il pourrait s'agir d'une inhibition de l'initiation florale dans un cas et d'une compétition pour les métabolites dans l'autre. L'absence de pollinisation entomophile modifie essentiellement le nombre de graines par silique et l'expression des phénomènes de compensation pour ce caractère puisque l'analyse de la variance des inflorescences secondaires montre qu'il n'y a pas de différence significative entre elles $(F=1,93$ pour 5 et 168 d.l.). La plante semble synthétiser suffisamment de métabolites pour nourrir toutes les graines formées, compte tenu de leur faible nombre et de la faible productivité de la hampe primaire. En revanche l'inhibition du développement en nombre de siliques de la $1^{\text {re }}$ secondaire se manifeste de façon identique dans les 2 lots.

Le nombre de graines par silique est le caractère qui traduit le mieux l'effet de la pollinisation puisqu'on obtient, sur les inflorescences d'ordre 5 et plus, des valeurs similaires à celles qui sont observées sur la hampe principale. Les variations entre inflorescences sont probablement dues au phénomène de compétition intra-plante plutôt qu'à une pollinisation différentielle par le bourdon qui aurait pu visiter préférentiellement les fleurs du haut de la plante comme le suggérait l'observation du comportement de ces insectes en plein champ.

Figure 1

Composantes du rendement par inflorescence.

Yield components for each raceme.

Sans bourdon (without bumblebees): symboles blancs (hollow symbols)

Avec bourdon (with bumblebees) : symboles noirs (black symbols) $\nabla \nabla$ nombre de siliques (number of pods);

- nombre de graines par silique (number of seeds per pod);

$\square$ poids d'une graine en $\mathrm{mg}$ (single seed weight in $\mathrm{mg}$ );

$I P=$ Inflorescences principales.

$I S_{1} \ldots I S_{5}=$ Inflorescences secondaires.

ISR $=$ Reste des inflorcscences secondaires (cf. texte).

Lc rendement de chaque inflorescence étant le produit des 3 composantes l'échelle logarithmique permet de le calculer par simple addition.

N. B. L'apparente dépression en IS $_{2}$ du lot non pollinisé provient d'un hasard d'échantillonnage, l'une des 2 cages ayant un nombre de graines par silique anormalement bas pour cette inflorescence. Ceci est confirmé par l'étude du nombre de graines par silique cn $I_{2}$ de 2 cages supplémentaires provenant du reste du dispositif expérimental (cf. chapitre IIIC). 


\section{Plantes entières (tabl. $4,5,6$ )}

Compte tenu de l'importance des inflorescences secondaires dans la production totale de la plante $(80,8 \mathrm{p} .100$ dans le lot non pollinisé et $71,6 \mathrm{p}, 100$ dans le lot pollinisé), les résultats concernant la plante entière s'apparentent à ceux des inflorescences secondaires. Les rendements des 2 lots ne sont pas significativement différents. La comparaison des diamètres au collet montre d'ailleurs que les 2 ensembles de plantes avaient a priori le même potentiel de production (VON SCHÜTTE, 1966) (tabl. 4).

Si l'on sélectionne les plantes des 2 lots ayant entre 7 et 11 inflorescences, bornes incluses, on a la totalité du lot pollinisé et 20 plantes du lot non pollinisé. Les différences entre les nombres totaux de siliques par plante disparaissent mais les rendements deviennent significativement différents (tabl. 5). Autrement dit, à nombre d'inflorescences égal, la pollinisation a augmenté le rendement. La faible productivité des $1^{\text {re }}$ inflorescences tend à être compensée par l'initiation d'inflorescences et de siliques supplémentaires mais ce phénomène ne touche qu'une partie des plantes. Un des effets les plus remarquables de la pollinisation est la diminution d'un facteur 4 de la variance du nombre de siliques par plante. Les cages utilisées faisaient partie d'un dispositif expérimental plus large destiné à étudier les pertes de rendement dues à un ravageur (Ceuthorrhynchus assimilis Payk.) avec et sans pollinisateur. L'effet du charançon, à densité faible, étant négligeable par rapport à l'effet de la pollinisation, les résultats de ces cages ont été joints à ceux discutés ci-dessus pour confirmer, sur un plus grand nombre de plantes, les résultats obtenus. Les résultats les plus représentatifs sont regroupés dans le tableau 6.

\section{E. Comparaison avec des plantes prélevées au champ (tabl. 7)}

Les semis en caissette ont été faits le jour du semis au champ. La phénologie des plantes a été assez semblable malgré un plus fort développement des plantes en caissette : la date d'ouverture de la $1^{\text {re }}$ fleur notamment (20 avril 1980) et celle de la fin de la floraison dans le lot pollinisé ( 25 mai 1980) ont été identiques dans les 2 cas. Sur l'ensemble des prélèvements au champ on a sélectionné les plantes ayant au moins 6 inflorescences productives $(n=35)$ et comparé les estimations du nombre de graines par silique (EG/Sx) sur les inflorescences primaires et sur la moyenne des inflorescences secondaires. La sélection des plantes les plus productives du champ est rendue nécessaire par la corrélation positive existant entre vigueur de la plante (mesurée par le nombre de siliques) et le nombre de graines par silique.

Les différences ne sont pas significatives, surtout si l'on tient compte du fait que le nombre d'inflorescences moyen est de 8,9 pour les plantes en cage et seulement 6,1 pour les plantes au champ: les plantes au champ, soumises à la compétition étaient moins vigoureuses que les plantes en caissette et l'avortement physiologique sur les inflorescences secondaires est plus important.

\section{TABLEAU 4}

Caractéristiques des plantes entières.

Characteristics of whole plants.

\begin{tabular}{|c|c|c|c|c|c|c|c|}
\hline & & $\mathrm{Nb}$ siliques & Graines/siliques & $\begin{array}{c}\text { Pds d'1 graine } \\
\text { (g) }\end{array}$ & $\begin{array}{l}\text { Rendement } \\
(\mathrm{g})\end{array}$ & $\begin{array}{l}\text { Diamètre } \\
\text { collet } \\
(\mathrm{cm})\end{array}$ & $\begin{array}{l}\text { Nb d'inflo- } \\
\text { rescences }\end{array}$ \\
\hline sans bourdon & $\begin{array}{l}\text { moy. } \\
\text { var. }\end{array}$ & $\begin{array}{l}336,4 \\
0,2492\end{array}$ & $\begin{array}{c}10,34 \\
0,0666\end{array}$ & $\begin{array}{c}6,43 \cdot 10^{-3} \\
0,0111\end{array}$ & $\begin{array}{l}22,38 \\
0,3376\end{array}$ & $\begin{array}{l}1,741 \\
0,0461\end{array}$ & $\begin{array}{r}10,17 \\
4,22\end{array}$ \\
\hline avec bourdons & $\begin{array}{l}\text { moy. } \\
\text { var. }\end{array}$ & $\begin{array}{l}278,0 \\
0,0642\end{array}$ & $\begin{array}{c}15,89 \\
0,0733\end{array}$ & $\begin{array}{c}6,13 \cdot 10^{-3} \\
0,0056\end{array}$ & $\begin{array}{c}28,15 \\
0,1061\end{array}$ & $\begin{array}{l}1,746 \\
0,0624\end{array}$ & $\begin{array}{l}8,93 \\
1,66\end{array}$ \\
\hline $\begin{array}{l}t \text { ou } t^{\prime} \\
\text { signification }\end{array}$ & & $\begin{array}{l}\text { (') } 1,846 \\
\mathrm{NS}\end{array}$ & $\begin{array}{c}6,293 \\
* *\end{array}$ & $\begin{array}{ll}\text { (') } & 2,032 \\
& \text { NS }\end{array}$ & $\begin{array}{l}\text { () } 1,869 \\
\text { NS }\end{array}$ & $\begin{array}{c}0,083 \\
\text { NS }\end{array}$ & (') $\begin{array}{l}2,770 \\
*\end{array}$ \\
\hline
\end{tabular}

TABLEAU 5

Comparaison des plantes ayant entre 7 et 11 inflorescences. Comparison between plants with 7 to 11 racemes.

\begin{tabular}{|c|c|c|c|c|c|c|c|}
\hline & & $\mathrm{Nb}$ siliques & Graines/siliques & $\begin{array}{c}\text { Pds d'1 graine } \\
(\mathrm{g})\end{array}$ & $\begin{array}{c}\text { Rendement } \\
\text { (g) }\end{array}$ & $\begin{array}{c}\text { Diamètre } \\
\text { collet } \\
(\mathrm{cm})\end{array}$ & $\begin{array}{l}\mathrm{Nb} \text { d'inflo- } \\
\text { rescences }\end{array}$ \\
\hline sans bourdon & $\begin{array}{l}\text { moy. } \\
\text { var. }\end{array}$ & $\begin{array}{r}279,3 \\
0,1623\end{array}$ & $\begin{array}{l}10,01 \\
0,0834\end{array}$ & $\begin{array}{c}6,67 \cdot 10^{-3} \\
0,0046\end{array}$ & $\begin{array}{l}18,63 \\
0,3257\end{array}$ & $\begin{array}{l}1,735 \\
0,0534\end{array}$ & $\begin{array}{l}9,00 \\
1,16\end{array}$ \\
\hline avec bourdon & $\begin{array}{l}\text { moy. } \\
\text { var. }\end{array}$ & $\begin{array}{l}276,6 \\
0,0628\end{array}$ & $\begin{array}{l}16,58 \\
0,0423\end{array}$ & $\begin{array}{c}6,11 \cdot 10^{-3} \\
0,0057\end{array}$ & $\begin{array}{l}28,04 \\
0,1006\end{array}$ & $\begin{array}{l}1,741 \\
0,0624\end{array}$ & $\begin{array}{l}8,90 \\
1,64\end{array}$ \\
\hline $\begin{array}{l}t \text { ou } t^{\prime} \\
\text { signification }\end{array}$ & & 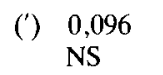 & $\begin{array}{c}6,787 \\
* *\end{array}$ & $\begin{array}{c}4,252 \\
* *\end{array}$ & (') $\underset{* *}{2,925}$ & $\begin{array}{c}0,298 \\
\text { NS }\end{array}$ & $\begin{array}{l}0,088 \\
\text { NS }\end{array}$ \\
\hline
\end{tabular}


TABLEAU 6

Comparaison entre plantes sur l'ensemble du dispositif expérimental (cf. texte).

Comparison between plants from the whole experiment (see text).

\begin{tabular}{|c|c|c|c|c|c|c|c|}
\hline & \multicolumn{3}{|c|}{ Inflorescences principales } & \multicolumn{4}{|c|}{ Plantes entières } \\
\hline & $\mathrm{Nb}$ siliques & $\begin{array}{l}\text { Longueur } \\
\text { inflorescence } \\
(\mathrm{cm})\end{array}$ & $\begin{array}{l}\text { Rendement } \\
(\mathrm{g})\end{array}$ & $\mathrm{Nb}$ siliques & $\begin{array}{l}\text { Rendement } \\
\text { (g) }\end{array}$ & $\begin{array}{l}\text { Diamètrc } \\
\text { collet } \\
\text { (cm) }\end{array}$ & $\begin{array}{l}\mathrm{Nb} \text { inflo- } \\
\text { rescences }\end{array}$ \\
\hline $\begin{array}{l}\text { sans bourdon } \\
\mathrm{n}=57\end{array}$ & 55,9 & 58,2 & 3,99 & 321,9 & 22,6 & 1,75 & 10,23 \\
\hline $\begin{array}{l}\text { avec bourdon } \\
\mathbf{n}=91\end{array}$ & 64,5 & 46,9 & 7,25 & 290,8 & 27,9 & 1,83 & 9,37 \\
\hline Signification & $* *$ & $* *$ & $* *$ & NS & * & NS & ** \\
\hline
\end{tabular}

N.B. : le pourcentage d'attaque moyen des siliques des deux lots par le ravageur est respectivement de 10,6 $\pm 3,0$ (lot non pollinisé) et $10,7 \pm 2,6$ (lot pollinisé).

\section{TABLEAU 7}

Comparaison des estimations du nombre de graines par silique au champ et en cage avec bourdon.

Comparison between estimations of number of seeds per pod in the field and in cages with bumble-bees.

\begin{tabular}{cccc}
\hline \hline & & $\begin{array}{c}\text { Inflorescences } \\
\text { principales }\end{array}$ & $\begin{array}{c}\text { Inflorescences } \\
\text { secondaires }\end{array}$ \\
\hline champ $\mathrm{n}=35$ & $\begin{array}{c}\text { moy. } \\
\text { var. }\end{array}$ & $\begin{array}{c}22,91 \\
0,0387\end{array}$ & $\begin{array}{c}16,32 \\
0,0786\end{array}$ \\
\hline cage $\mathrm{n}=31$ & moy. & $\begin{array}{c}21,47 \\
0,0427\end{array}$ & $\begin{array}{c}18,50 \\
0,0618\end{array}$ \\
\hline var. & & 1,300 & 1,921 \\
signification & & $\mathrm{NS}$ & $\mathrm{NS}$ \\
\hline
\end{tabular}

$\mathrm{n}=$ nombre de plantes examinées.

\section{CONCLUSION}

Cette expérience a été entreprise dans un but méthodologique pour définir les conditions dans lesquelles le rendement et les composantes du rendement présentent un minimum de variations entre plantes ; ceci afin de faciliter la mise en évidence des pertes de production dues au charançon des siliques ou d'autres ravageurs.

Sauf circonstances exceptionnelles, un champ de colza est visité par de nombreux pollinisateurs (abeilles domestiques, andrènes, bourdons) qui assurent une bonne pollinisation.
Les ravageurs floricoles du type méligèthes pourraient également, vu leur nombre, avoir un rôle non négligeable sur le transfert du pollen des étamines au style (VON HÄRLE, 1948). D'autre part, le vent qui agite le colza et transporte le pollen est toujours présent dans la nature.

La mise sous cage, en limitant l'effet du vent et en soustrayant les fleurs à la visite des pollinisateurs, entraîne un déficit de pollinisation et une certaine hétérogénéité dans le développement des plantes, comme en témoigne l'augmentation des variances associées aux variables mesurées. L'augmentation de $26 \mathrm{p} .100 \mathrm{du}$ rendement du lot pollinisé par rapport au lot non pollinisé n'est pas significative. En définitive, l'effet majeur de la pollinisation est de limiter les variations des composantes du rendement et d'homogénéiser la production. Il y a là essentiellement un avantage qualitatif : très forte augmentation de la production de l'inflorescence principale, réduction du nombre d'inflorescences, notamment tertiaires, ce qui contribue à une maturation plus rapide et plus homogène. Les plantes pollinisées sont également plus représentatives des plantes au champ les plus productives, du moins en ce qui concerne le nombre de graines par silique. En conséquence, toute étude sous cage à mailles fines avec des plantes peu serrées ou isolées devrait comporter l'adjonction d'un pollinisateur, surtout lorsqu'on cherche à réduire au maximum les variations entre plantes. L'utilisation de bourdons prélevés dans la nature, avant fondation des colonies, est une méthode pratique pour assurer une bonne pollinisation. Il faut cependant que les plantes soient assez nombreuses pour que les pollinisateurs disposent d'une alimentation suffisante, nécessaire à une activité soutenue.

Reçu le 28 septembre 1981. Accepté le 10 novembre 1981. 


\section{RÉFÉRENCES BIBLIOGRAPHIQUES}

Barbier E., 1978. Pollinisation du colza par les abcilles. Rev. fr. d'Apic. 365, 288-291.

Benedek P., Komlódi J., Prenner J., Wilhem E., 1972. Insect pollination of oilseed rape (Brassica napus L.). Növenytermeles, 21 (3), 255-269 (en hongrois).

Brar G., Thies W., 1977. Contribution of leaves, stem, siliques and seeds to dry matter accumulation in ripening seeds of rapeseed, Brassica napus L. Z. Pflanzenphysiol., 82, 1-13.

Clarke J. M., 1979. Intraplant variation in number of seeds per pod and seed weight in Brassica napus « Tower ». Can. J. Plant Sci., 59, 959-962.

Eisikowitch D., 1981. Some aspects of pollination of oil-seed rape (Brassica napus L.). J. agric. Sci. Camb., 96, 321-326.

Free J. B., Nuttall P. H., 1968. The pollination of oilseed rape (Brassica napus) and the behaviour of bees on the crop. J. agric. Sci. Camb., 71, 91-94.
Härle A. Von, 1948. Ist der Rapsglanzkäfer (Meligethes aeneus Fabr.) nur ein Schädling. Nachrichtenbl. dtsch. Pflanzenschutzdienst, 2, 40-42.

Mesquida J., Renard M. Pollinisation du colza d'hiver mâle-fertile et mâle-stérile (Brassica napus L. var. oleifera Metzger) par l'abeille domestique (Apis m. mellifica L.). Effets sur la phénologie et le rendement. Apidologie (sous presse).

Rives M., 1957. Etudes sur la sélection du colza d'hiver. Ann. Amélior. Plant., 1, 61-107.

Schütte F. Von, 1966. Ein einfaches Verfahren zur Ermittlung des Schadens der Kohlschoten mücke (Dasyneura brassicae Winn.) und zur Schätzung der Rapsernte. Anz. Schadlingskd. Pflanzen. Umweltsch., 39 (11), 167-171.

Williams I. M., 1978. The pollinisation requirements of swede rape (Brassica napus L.) and of turnip rape (Brassica campestris L.). J. agric. Sci. Camb., 91, 343-348. 